\title{
A ruptured large aneurysm of the ductus arteriosus
}

\author{
SHOJI TSUJIMOTO, KUNIHIKO HIROSE, AKIRA OHYAGI \\ From the Cardiovascular Department, Ohtsu Red-Cross Hospital, Nagara, Ohtsu City, Fapan
}

SUMMARY A 60 year old man was admitted with acute back pain followed by hoarseness. An aneurysm of the ductus arteriosus Botalli was diagnosed and an operation was recommended because of the high risk of complications such as rupture, embolism, or infection. The patient and his family refused surgery, however. The patient died suddenly of a rupture of the aneurysm a year later; necropsy confirmed the diagnosis. Several diagnostic methods were used and enhanced computed tomography gave the best representation of the aneurysm as it was seen at necropsy.

This case indicates that enhanced computed tomography is probably the most useful investigation in patients with this type of aneurysm and it confirms the importance of an aneurysmectomy.

An aneurysm of the ductus arteriosus Botalli is an uncommon cardiovascular anomaly. Cases in adults are rare; only 22 had been reported by $1984 .^{12} \mathrm{We}$ report a case of an aneurysm of a non-patent ductus arteriosus in an adult. In this case the diagnosis was made clinically but surgery was refused and death occurred suddenly about one year after diagnosis. Necropsy showed a left haemothorax caused by a ruptured aneurysm of a non-patent ductus arteriosus. This case raises several important issuessuch as the most useful diagnostic methods and the importance of surgery.

\section{Case report}

A 60 year old man was admitted with acute severe back pain followed by hoarseness in January 1983. Five days before admission he had had acute back pain lasting all day. He visited the otolaryngological department of our hospital because of hoarseness, and left recurrent nerve paralysis was diagnosed. He was referred to the cardiovascular department because of an abnormally large aortic shadow on the chest $x$ ray. He had a 10 year history of diabetes mellitus and hypertension, which were well controlled. There was no history of chest trauma.

At admission blood pressure was $142 / 90 \mathrm{~mm} \mathrm{Hg}$ and a grade $3 / 6$ mid-systolic murmur was audible at the pulmonary area (the second intercostal space

Requests for reprints to Dr Akira Ohyagi, Cardiovascular Department, Kyoto Katsura Hospital, 17 Hirao-Cho Yamada Nishikyo-Ku, Kyoto City, Zip 615 Japan. along the left sternal border). A chest $x$ ray showed a smooth rounded dense area in the left hilar region that was contiguous with the aorta, reduced lung markings throughout the left lung, and a cardiothoracic ratio of 0.54 . The electrocardiogram disclosed right ventricular hypertrophy (fig 1). A serological test for syphilis was negative. An enhanced computed tomogram of the chest showed a large aneurysm between the ascending aorta and the descending aorta that contained a mural thrombus (fig 2). The maximum transverse diameter of the aneurysm was $118 \mathrm{~mm}$. A pulmonary scintigram showed absence of left lung perfusion. Right heart catheterisation showed mild elevation of right ventricular pressure $(51 \mathrm{~mm} \mathrm{Hg}$ systolic $/ 6 \mathrm{~mm} \mathrm{Hg}$ end diastolic) and a systolic pressure gradient of $25 \mathrm{~mm} \mathrm{Hg}$ between the main $(52 / 6 \mathrm{~mm} \mathrm{Hg})$ and right pulmonary arteries $(27 / 8 \mathrm{~mm} \mathrm{Hg})$. This systolic pressure gradient was thought to be the cause of the heart murmur in this patient. Pulmonary arteriography showed that the left pulmonary artery was completely obstructed at its origin. The laevophase of the same arteriogram showed a large aortic aneurysm protruding from the inferior border of the aortic arch.

Because of the high risk of fatal complications such as rupture, embolism, and infection, operation was recommended, but the patient and his family refused it and he was discharged. He was treated medically to reduce afterload. On 13 December 1983 the patient again presented with severe chest pain, which lasted until he died early the next morning.

Necropsy showed approximately 2 litres of bloody 


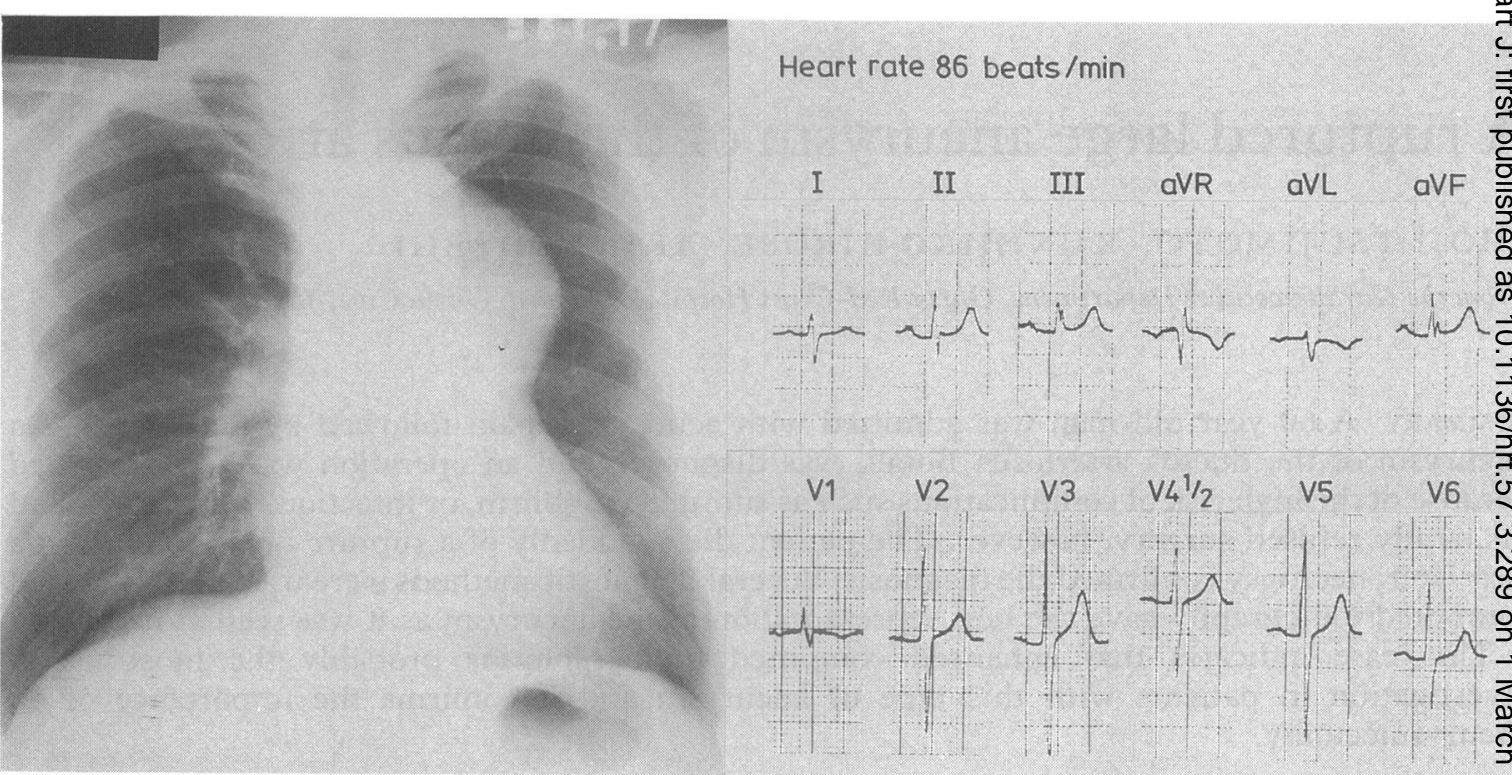

Fig 1 Chest radiogram (left) and electrocardiogram (right) on admission. There is a smooth rounded dense area in the left hilar region contiguous with the aorta and reduced lung markings throughout the left lung. The electrocardiogram disclosed . right ventricular hypertrophy.

pleural fluid in the left pleural cavity and a ruptured large thoracic aneurysm surrounded by the aortic arch. The aortic ostium of the aneurysm was patent and was located at the site of the ductus arteriosus Botalli. The pulmonary end of the aneurysm was closed. This ligamentum arteriosus was not identified because of severe adhesions between the main pulmonary artery and the aneurysm. Most of

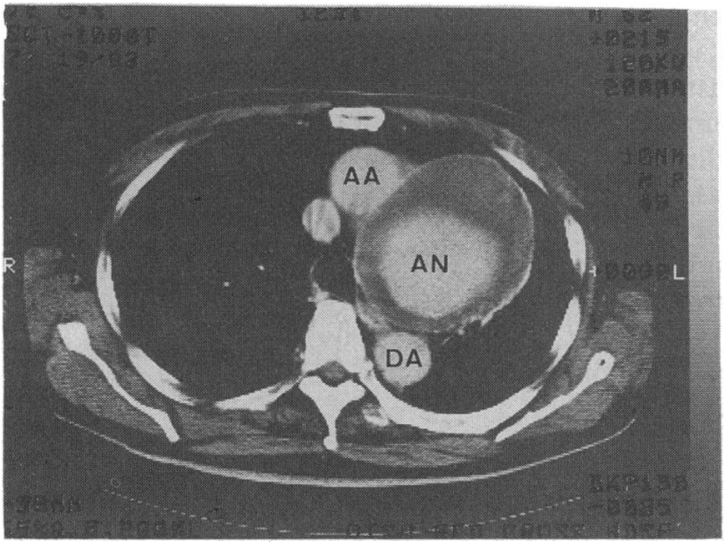

Fig 2 Enhanced computed tomogram of the chest. A large aneurysm is located between the ascending aorta and the descending aorta. Most of the aneurysmal cavity is occupied by a mural thrombus. $A A$, ascending aorta; $D A$, descending aorta; $A N$, aneurysm. its cavity was occluded by a mural thrombus a only the central part of the aneurysm was patent. The aneurysm protruded anteriorly and to the lest. There was a rupture $5 \mathrm{~cm}$ long on the anteroinferier wall of the aneurysm. The aneurysm completey compressed the left pulmonary artery and moderately compressed the right pulmonary artery. The right ventricle showed concentric hypertrophy; its wall was $4 \mathrm{~mm}$ thick. There was severe atherosclerosis with calcification in the aorta.

\section{Discussion}

Aneurysms originating from the ductus arteriosws Botalli are called ductal aneurysms. When the aor ic ostium of the duct fails to close the systemic blopd pressure in the aorta may lead to aneurysmal dilatation of the duct. ${ }^{2-4}$

Aneurysm of the ductus arteriosus Botalli vês first described by Martin et al in $1827^{6}$; only 22 ad बit cases had been reported by 1984 . Although "the prognosis in the majority of infantile ductus arteriosus aneurysms without treatment is good", 5 ? : adults rupture, embolism, or infection are comm fatal complications. Mitchell et al noted that $60 \% \%$ f reported patients died of complications related ao the aneurysm. ${ }^{1}$ Operation is recommended $\mathrm{B}$ aneurysms that are $>3 \mathrm{~cm}$ in diameter, if sympto indicate that an adjacent organ has been affected by 
the aneurysm, or if there is progressive enlargement of the aneurysm. ${ }^{135}$ In our case we advised operation but unfortunately the patient and his family refused. The patient died a year after diagnosis of the aneurysm.

This case indicates that operation may be mandatory. A correct diagnosis is essential especially before operation. In our patient enhanced computed tomography gave the most reliable information and was as useful as invasive examinations such as angiocardiography. Until now angiocardiography has been considered to be one of the most reliable tools for the diagnosis of aneurysms, although there is a risk of rupturing the aneurysm when contrast medium is injected into the aorta under high pressure. We recommend enhanced computed tomography as a very useful tool for diagnosis of aneurysm of the ductus arteriosus. It should lead to "the correct preoperative diagnosis and the proper surgical management". 4

We thank Dr Alice S Cary, and Dr Yasuyuki
Nakamura for their help in the preparation of this paper.

\section{References}

1 Mitchell RS, Seifert FC, Miller DC, Jamieson SW, Shumway NE. Aneurysm of the diverticulum of the ductus arteriosus in the adult. $f$ Thorac Cardiovasc Surg 1983;86:400-8.

2 Danza FM, Fusco A, Breda M, Bock E, Lemmo G, Colavita N. Ductus arteriosus aneurysm in an adult. Am f Roentgenol 1984;143:131-3.

3 Falcone MW, Perloff JK, Roberts WC. Aneurysm of the nonpatent ductus arteriosus. Am $\mathcal{f}$ Cardiol 1972;29:422-6.

4 Cohen BA, Efremidis SC, Dan SJ, Robinson B, Rabinowitz JG. Aneurysm of the ductus arteriosus in an adult. $\mathcal{F}$ Comput Assist Tomogr 1981;5:421-3.

5 Heikkinen ES, Simila S, Laitinen J, Larmi T. Infantile aneurysm of the ductus arteriosus. Acta Paediatr Scand 1974;63:241-8.

6 Martin. Dilatation anéurysmal du canal arteriel. Bull Soc Anat Paris 1827;22:17. 